where $a$ and $b$ are as defined in the statement of the theorem. From this (4.1) now follows, and the theorem is proved.

Examples. If $a_{2}=2+3 i / 2, a_{3}=-i / 2, a_{4}=-1 / 4$ we find that

$$
\left|w-\frac{24(78-53 i)}{8893}\right| \leqq \frac{240}{8893} \text {. }
$$

If $a_{2}=1 / 2, a_{3}=1 / 6, a_{4}=1 / 3$, we find that

$$
|w-.69375| \leqq .00625 \text {. }
$$

In this case the first four partial quotients are the same as those in a continued fraction for $\log 2=.6931 \cdots$.

NORTHWESTERN UNIVERSITY

\title{
SUR LE NOMBRE COMPLEXE BINAIRE
}

\section{PEDRO F. CAPELLI}

\section{INTRODUCTION}

L'objet de ce travail est de démontrer que la théorie des fonctions polygènes ${ }^{1} d^{\prime}$ 'un variable complexe connu et celles d'un variable complexe duel et hyperbolique développées par les docteurs Vignaux et Durañona y $\mathrm{Vedia}^{2}$ sont des cas particuliers d'une même théorie qui en contient d'autres.

On doit l'origine de cette théorie à la suivante interprétation géométrique de la définition d'unité imaginaire $i^{2}=-1, j^{2}=1, k^{2}=0$.

Si nous considérons le point représentatif de cette unité, nous observons que son carré représente un autre point qui géométriquement signifie, dans le champ complexe ordinaire, une rotation de $+\pi / 2$, et, dans le champ complexe hyperbolique, une rotation de $-\pi / 2$ et dans le duel une translation à zéro.

Il n'y a rien de plus naturel que de considérer ces questions comme cas particuliers d'une rotation et d'une translation combinées, c'est-àdire que nous mettons un complexe $a+b \alpha$ dont l'unité imaginaire $\alpha$ est telle que $\alpha^{2}=\mu+\nu \alpha$, ce qui analytiquement exprime le concept géométrique que nous venons de dire. Quand

\footnotetext{
${ }^{1}$ Le mot polygène était introduit par E. Kasner. Voyez son premier papier: $A$ new theory of polygenic or nonmonogenic functions, Science, vol. 66 (1927), pp. 581-582.

${ }^{2}$ Sobre las funciones de una variable compleja hiperbólica, Contribución al Estudio de las Ciencias Físicomatemáticas, vol. 1, estudio $2^{\text {a }}$, 1935. J. C. Vignaux, Sobre la teoria de fonciones poligenas de una y varias variables complejas duales, ibid., estudio $3^{\mathrm{a}}$.
} 
$\nu=0, \mu=-1$ nous avons le complexe ordinaire, $\nu=0, \mu=1$, nous avons le complexe hyperbolique, $\nu=0, \mu=0$, nous avons le complexe duel.

Alors on définit ainsi les opérations fondamentales: Égalité et addition comme dans le cas du variable complexe ordinaire. Produit

$$
(a, b) \times\left(a^{\prime}, b^{\prime}\right)=\left(a a^{\prime}+\mu b b^{\prime}, a^{\prime} b+a b^{\prime}+\nu b b^{\prime}\right) .
$$

Dans ce champ on pourrait entendre que le nombre $\mu$ joue le rôle de dilatation (puisque si $\nu=0$, on passe des complexes connus aux complexes généraux en multipliant par $\mu$ ) et $\nu$ joue le rôle de rotation puisque il transporte le point correspondant au carré de l'unité imaginaire du champ réel au complexe.

Les propriétés de ce champ sont caracterisées par le déterminant

$$
\Delta=\nu^{2}+4 \mu=\left|\begin{array}{rr}
\nu & \mu \\
-4 & \nu
\end{array}\right|
$$

Selon que $\Delta<0, \Delta=0, \Delta>0$ nous avons les complexes elliptiques, paraboliques ou hyperboliques, avec l'intéressante propriété que, avec un changement d'unités, ces champs peuvent être transformés respectivement en d'autres ayant la nouvelle unité $\alpha^{2}=-1,01$.

Géométriquement ce changement d'unités signifie une rotation de l'axe $y$ de sorte qu'il forme avec l'axe $x$ un angle $\omega$ donné par tg $\omega$ $=-2 / \nu$ et les formules de passage sont pour les champs elliptiques et hyperboliques $x=x^{\prime}+p \nu y^{\prime}, y=-2 p y^{\prime}$; et pour le champ parabolique

$$
x=x^{\prime}-\frac{\nu b}{2} y^{\prime}, \quad y=b y^{\prime}
$$

$p$ étant une constante qui dépend du champ.

Nous introduisons le concept de distance binaire qu'on exprime pour un complexe $a+b \alpha$ à l'égard de l'origine des coordonnées

$$
\left(\left|a^{2}+\nu a b-\mu b^{2}\right|\right)^{1 / 2} \text {. }
$$

Cette forme est invariable à l'égard des mouvements de la géométrie qui correspond à ces complexes et elle est aussi égale à la racine carrée du produit de deux complexes symétriques que nous définissons de la façon suivante: $a+b \alpha,(a+\nu b)-b \alpha$.

Cette formule (1) nous permet d'introduire le concept de module, de manière qu'on peut conserver la loi du module du produit des complexes, comme nous allons voir en introduisant l'expression trigonométrique des nombres de ce champ. 
A ce propos nous introduisons le concept généralisé de fonctions trigonométriques, avec la définition suivante.

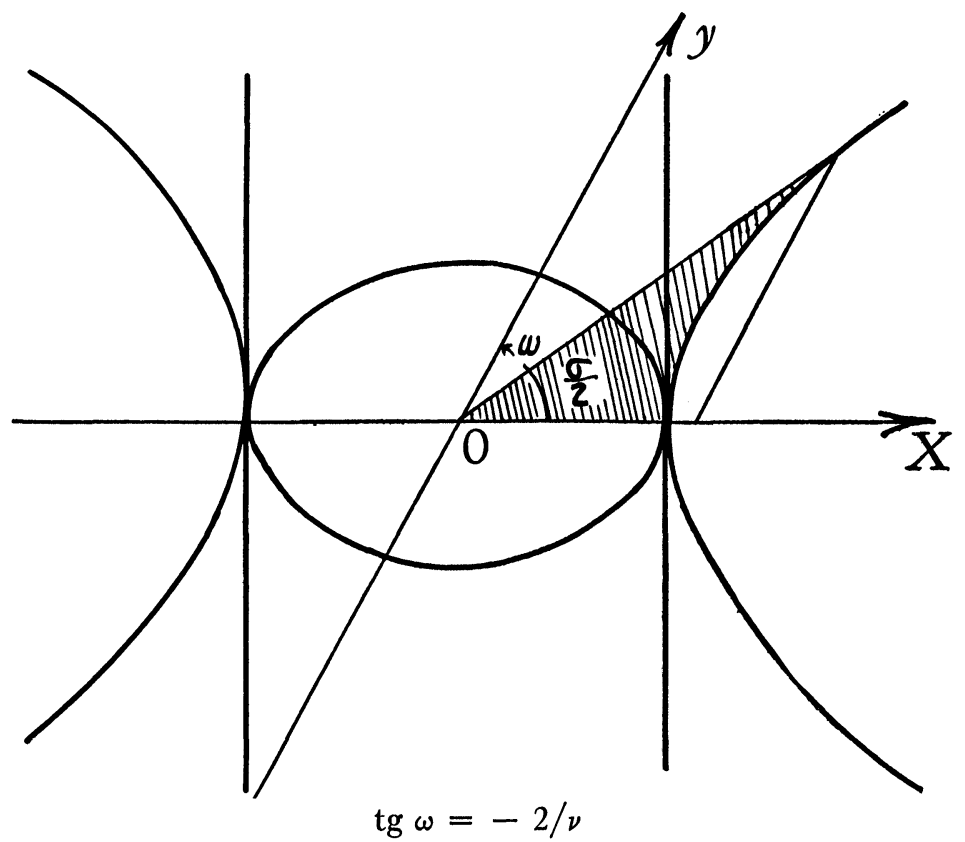

Nous considérons une conique avec centre, une ellipse pour le champ complexe ordinaire, une paire de droites parallèles pour le champ complexe parabolique et une hyperbole pour le champ complexe hyperbolique référées aux axes indiquées dans la figure.

En suivant la définition classique nous appellerons argument $\sigma$ l'aire déterminée par la conique comme indiqué dans la figure, et nous appellerons $\sin \sigma(\cos \sigma)$ l'ordonnée (abcisse) du point de la conique déterminé par l'intersection avec le rayon vecteur et $\operatorname{tg} \sigma$ le quotient du $\sin \sigma$ et $\cos \sigma$.

On déduit à l'égard de ces définitions, entre autres, les formules suivantes:

$$
\begin{aligned}
& \sin \left(\sigma_{1}+\sigma_{2}\right)=\cos \sigma_{1} \sin \sigma_{2}+\cos \sigma_{2} \sin \sigma_{1}+\nu \sin \sigma_{1} \sin \sigma_{2}, \\
& \cos \left(\sigma_{1}+\sigma_{2}\right)=\cos \sigma_{1} \cos \sigma_{2}+\mu \sin \sigma_{1} \sin \sigma_{2}, \\
& \sin \left(-\sigma_{1}\right)=-\sin \sigma_{1}, \quad \cos (-\sigma)=\cos \sigma+\nu \sin \sigma \\
& \operatorname{tg}\left(\sigma_{1}+\sigma_{2}\right)=\frac{\operatorname{tg} \sigma_{1}+\operatorname{tg} \sigma_{2}+\nu \operatorname{tg} \sigma_{1} \operatorname{tg} \sigma_{2}}{1+\mu \operatorname{tg} \sigma_{1} \operatorname{tg} \sigma_{2}}, \\
& \cos ^{2} \sigma+\nu \cos \sigma \sin \sigma-\mu \sin ^{2} \sigma=1 .
\end{aligned}
$$


Ces formules contiennent comme cas particuliers les formules trigonométriques circulaires, hyperboliques et le duel introduit par le Dr. Vignaux.

En outre on trouve une généralisation des formules d'Euler:

$$
\sin \sigma=\frac{e^{\alpha \sigma}-e^{-\alpha \sigma}}{2 \alpha-\nu} ; \quad \cos \sigma=\frac{\alpha\left(e^{\alpha \sigma}+e^{-\alpha \sigma}\right)-\nu e^{\alpha \sigma}}{2 \alpha-\nu} .
$$

Nous pouvons exprimer, maintenant, le nombre complexe binaire en forme trigonométrique $\rho(\cos \sigma+\alpha \sin \sigma)$ et on conserve dans cette formule les lois du produit, quotient, la formule de Moivre, et cetera.

Comme résultat de la définition de distance, on étend immédiatement le concept de voisinage, qui devient une ellipse, une paire de droites parallèles ou une hyperbole dont le centre est le point donné, respectivement, pour les champs complexe elliptique, parabolique ou hyperbolique.

Il est très important de remarquer que les asymptotes de ces coniques qui déterminent le voisinage du point zéro sont le lieu géométrique des diviseurs de zéro; ensuite de notre point de vue général les complexes elliptiques ont aussi des diviseurs de zéro, mais situés sur deux droites de pente imaginaire, qui sont les asymptotes imaginaires de l'ellipse.

Si le voisinage est conçu de cette façon, nous avons alors d'autres considérations que nous exprimons tout de suite.

Quand le rayon s'approche à zéro, la conique tend à se confondre avec ses asymptotes. Cette circonstance ainsi que celle où les diviseurs de zéro occupent les asymptotes, c'est-à-dire que la distance binaire entre ses points quelconques est zéro, donnent lieu à un intéressant concept de point, en le concevant comme un système formé par un point dans le sens ordinaire et la paire de droites parallèles aux droites isotropes (droites des diviseurs de zéro) du champ, qui passent par ce point.

De cette façon nous obtiendrons une image géométrique des points que nous appellerons point elliptique, point parabolique et point hyperbolique, selon le cas.

Nous considerons deux plans, l'un supérieur et l'autre inférieur, que nous imaginons unis comme des plans ponctuels, mais non pas comme des plans réglés, en exceptant une direction.

Alors le point elliptique sera répresenté par un point et la paire de droites parallèles aux droites isotropes qui passent par ce point dans le plan inférieur; le point parabolique sera répresenté par un point et la paire de droites coincidentes selon la direction commune aux plans; 
et point hyperbolique sera le point et les droites parallèles aux isotropes qui passent par ce point dans le plan supérieur.

De cette façon le plan inférieur contient les droites imaginaires et le supérieur les droites réelles.

En outre on peut passer, par continuité, en tournant autour d'un point, du plan supérieur à l'inférieur et réciproquement.

A ce passage géométrique continu on fait correspondre la variation continue du déterminant du champ, $\Delta=\nu^{2}+4 \mu$, de valeurs positives à negatives.

\section{Fonctions Polygìnes DE VARIABLE COMPLEXE BINAIRE}

Les concepts de variable complexe, fonction, limite et continuité sont les classiques.

Dérivé radial. Soit la fonction de variable complexe binaire $w=f(z)=u(x y)+v(x y) \alpha$ où $z=x+y \alpha\left(\alpha^{2}=\mu+\nu \alpha\right)$ et considérons le rapport $\left[f(z)-f\left(z_{0}\right)\right] /\left[z-z_{0}\right]$ avec $z$ et $z_{0}$ points d'un domaine $D$ de la fonction, avec $z_{0}$ fixe et $z$ variable de telle façon qu'il n'appartient pas aux droites isotropes qui passent par $z_{0}$.

Aussi nous avons

$$
f(z)-f\left(z_{0}\right)=\Delta w=\Delta u+\Delta v \cdot \alpha, \quad z-z_{0}=\Delta z=\Delta x+\Delta y \cdot \alpha
$$

et

$$
\frac{\Delta w}{\Delta z}=\frac{\Delta u+\Delta v \cdot \alpha}{\Delta x+\Delta y \cdot \alpha} .
$$

Si les fonctions $u$ et $v$ sont différentielles dans le sens de StolzFréchet il en résulte

$$
\begin{aligned}
& \Delta u=\frac{\partial u}{\partial x} \Delta x+\frac{\partial u}{\partial y} \Delta y+\epsilon_{1} \Delta x+\epsilon_{2} \Delta y, \\
& \Delta v=\frac{\partial v}{\partial x} \Delta x+\frac{\partial v}{\partial y} \Delta y+\epsilon_{1}^{\prime} \Delta x+\epsilon_{2}^{\prime} \Delta y,
\end{aligned}
$$

$\epsilon_{1}, \epsilon_{2}, \epsilon_{1}^{\prime}, \epsilon_{2}^{\prime}$ étant infiniment petits avec $\Delta y$ et $\Delta x$.

Substituant en (1), divisant par $\Delta x$ et passant au limite par $|\Delta z| \rightarrow 0$ on a

$$
\left(\frac{d w}{d z}\right)_{n}=\frac{\left(\frac{\partial u}{\partial x}+\frac{\partial v}{\partial x} \alpha\right)+n\left(\frac{\partial u}{\partial y}+\frac{\partial v}{\partial y} \alpha\right)}{1+n \alpha}=X+Y \alpha
$$

où $\Delta y / \Delta x=n$; expression que définit le dérivé radial selon la direc- 
tion $n$. On doit exclure les directions $(\nu \pm m) / 2 \mu$ qui correspondent aux droites des diviseurs de zéro.

Conditions de monogénéité. La fonction w sera monogène dans un point quand le dérivé est égal pour une direction quelconque. Nous pouvons trouver les conditions de monogénéité en applicant le théorème suivant. L'expression $(A+B n) /(C+D n)$, avec $A, B, C$ et $D$ complexes binaires et $n$ un paramètre réel, est indépendante de $n$ quand

$$
A D=B C,
$$

tandis que $C$ et $D$ ne sont pas des diviseurs de zéro et $n$ ne prennent pas deux valeurs qui fassent le dénominateur diviseur de zéro.

En applicant ce théorème à notre expression (2) où

$$
A=\frac{\partial u}{\partial x}+\frac{\partial v}{\partial x} \alpha, \quad B=\frac{\partial u}{\partial y}+\frac{\partial v}{\partial y} \alpha, \quad C=1, \quad D=\alpha
$$

il résulte, excepté les valeurs de $n=(\nu \pm m) / 2 \mu$, que la condition nécessaire et suffisante pour que $d w / d z$ soit indépendante de $n$ est que

$$
\left(\frac{\partial u}{\partial x}+\frac{\partial v}{\partial x} \alpha\right) \alpha=\frac{\partial u}{\partial y}+\frac{\partial v}{\partial y} \alpha
$$

qui exige

$$
\frac{\partial u}{\partial x}=\frac{\partial v}{\partial y}-\nu \frac{\partial v}{\partial x}, \quad \frac{\partial u}{\partial y}=\mu \frac{\partial v}{\partial x} .
$$

Ces conditions de monogénéité contiennent comme cas particuliers ces conditions de monogénéité pour les fonctions de variable complexe ordinaire, hyperbolique et duel.

En effet, quand

$$
\begin{aligned}
& \nu=0, \mu=-1, \partial u / \partial x=\partial v / \partial y \text { et } \partial v / \partial x=-\partial u / \partial y, \\
& \nu=0, \mu=1, \partial u / \partial x=\partial v / \partial y \text { et } \partial v / \partial x=\partial u / \partial y, \\
& \nu=0, \mu=0, \partial u / \partial x=\partial v / \partial y \text { et } \partial u / \partial y=0 .
\end{aligned}
$$

En admettant l'existence des dérivées seconds de $u$ et $v$, et si les théorèmes de permutabilité de la dérivation tiennent, on trouve les relations

$$
\frac{\partial^{2} u}{\partial y^{2}}-\mu \frac{\partial^{2} u}{\partial x^{2}}-\nu \frac{\partial^{2} u}{\partial x \partial y}=0, \quad \frac{\partial^{2} v}{\partial y^{2}}-\mu \frac{\partial^{2} v}{\partial x^{2}}-\nu \frac{\partial^{2} v}{\partial x \partial y}=0,
$$


équations qui contiennent comme cas particuliers celles de Laplace et celles déjà connues pour les variables complexes hyperboliques et duels.

Il en résulte, en outre, que la fonction holomorphe reste déterminée par ses valeurs de contour comme on démontre dans un travail publié.

Interprétation géométrique de le dérivé radial. L'expression de le dérivé radial

$$
\left(\frac{d w}{d z}\right)_{n}=\frac{\left(\frac{\partial u}{\partial x}+\frac{\partial v}{\partial x} \alpha\right)+n\left(\frac{\partial u}{\partial y}+\frac{\partial v}{\partial y} \alpha\right)}{1+n \alpha}=X+Y \alpha
$$

où $\Delta y / \Delta x=n$, admet une interprétation géométrique simple et intéressante. En effet, de la (3) il résulte

$$
\begin{aligned}
& X=\frac{u_{x}+m u_{y}+m u_{x} \nu+m^{2} u_{y} \nu-v_{x} m \mu-v_{y} m^{2} \mu}{m \nu-m^{2} \mu+1}, \\
& Y=\frac{v_{x}+m v_{y}-m u_{x}-m^{2} u_{y} u}{m \nu-m^{2} \mu+1} .
\end{aligned}
$$

Si nous représentons les variables $z=x+y \alpha, w=u+v \alpha$ dans deux plans respectivement et si nous adoptons un troisième plan pour représenter

$$
\left(\frac{d w}{d z}\right)_{n}=X+Y \alpha,
$$

à chaque point $z_{0}$ de $z$ correspond un point $w_{0}$ de $w$, tandis que pour le plan de $d w / d z$ il résulte des infinis points correspondants aux infinies valeurs que peuve prendre $n .^{3}$

Nous allons montrer maintenant que ces points déterminent une conique.

En effet, les relations (4) peuvent s'écrire

$$
X=\frac{\left|\begin{array}{cc}
u_{x}+n u_{y} & n \mu \\
v_{x}+n v_{y} & 1+n \nu
\end{array}\right|}{\left|\begin{array}{cc}
1 & n \mu \\
n & 1+n \nu
\end{array}\right|}, \quad Y=\frac{\left|\begin{array}{cc}
1 & u_{x}+n u_{y} \\
n & v_{x}+n v_{y}
\end{array}\right|}{\left|\begin{array}{cc}
1 & n \mu \\
n & 1+n \nu
\end{array}\right|}
$$

d'où il résulte que $X$ et $Y$ sont les racines du système.

\footnotetext{
${ }^{3}$ Kasner, A complete characterization of the derivative of a polygenic function, Pro-
} ceedings of the National Academy of Sciences, vol. 22 (1936), pp. 172-177. 


$$
X+n \mu Y=u_{x}+n u_{y}, \quad n X+(1+n v) Y=v_{x}+n v_{y} .
$$

Pour chaque valeur de $n$, la solution de ce système représente un point du plan et nous obtiendrons le lieu géométrique en faisant disparaître $n$ entre les deux. Alors il résulte que

$$
X^{2}-\mu Y^{2}+A Y-B X+\nu X Y+C=0,
$$

qui est la conique cherchée, avec

$$
\begin{gathered}
A=\frac{\partial y}{\partial y}-\nu \frac{\partial u}{\partial x}+\mu \frac{\partial v}{\partial x} ; \quad B=\frac{\partial u}{\partial x}+\frac{\partial v}{\partial y} ; \\
C=\frac{\partial u}{\partial x} \cdot \frac{\partial v}{\partial y}-\frac{\partial u}{\partial y} \cdot \frac{\partial v}{\partial x} .
\end{gathered}
$$

On a pour

$$
\begin{aligned}
& \nu^{2}+4 \mu>0, \text { la (5) est type hyperbole, } \\
& \nu^{2}+4 \mu=0, \text { la (5) est type parabole, } \\
& \nu^{2}+4 \mu<0, \text { la (5) est type ellipse. }
\end{aligned}
$$

En particulier pour $\nu=0, \mu=-1$, la (5) donne

$$
\left(X-A_{1}\right)^{2}+\left(Y-B_{1}\right)^{2}=R_{1}^{2}
$$

avec

$$
\begin{aligned}
& A_{1}=\frac{1}{2}\left(\frac{\partial u}{\partial x}+\frac{\partial v}{\partial y}\right) ; \quad B_{1}=\frac{1}{2}\left(\frac{\partial v}{\partial x}-\frac{\partial u}{\partial y}\right) ; \\
& R_{1}^{2}=\frac{1}{4}\left[\left(\frac{\partial u}{\partial x}-\frac{\partial v}{\partial y}\right)^{2}+\left(\frac{\partial v}{\partial x}+\frac{\partial u}{\partial y}\right)^{2}\right]
\end{aligned}
$$

qui est le cercle de Kasner. ${ }^{4}$ Pour $\nu=0, \mu=1$, la (5) donne

$$
\left(X-A_{2}\right)^{2}-\left(Y-B_{2}\right)^{2}=R_{2}^{2}
$$

avec

$$
\begin{aligned}
& A_{2}=\frac{1}{2}\left(\frac{\partial u}{\partial x}+\frac{\partial v}{\partial y}\right) ; \quad B_{2}=\frac{1}{2}\left(\frac{\partial u}{\partial y}+\frac{\partial v}{\partial x}\right) ; \\
& R_{2}^{2}=\frac{1}{4}\left[\left(\frac{\partial u}{\partial x}+\frac{\partial v}{\partial y}\right)^{2}-\left(\frac{\partial u}{\partial y}-\frac{\partial v}{\partial x}\right)^{2}\right]
\end{aligned}
$$

qui est la hyperbole dérivée. Pour $\nu=0, \mu=0$, la (5) donne

${ }^{4}$ Kasner and De Cicco, The derivative circular congruence-representation of a polygenic function, American Journal of Mathematics, vol. 61 (1939), pp. 995-1003. 
(8) $X^{2}+\frac{\partial u}{\partial y} Y-\left(\frac{\partial u}{\partial x}+\frac{\partial v}{\partial y}\right) X+\left(\frac{\partial u}{\partial x} \cdot \frac{\partial v}{\partial y}-\frac{\partial u}{\partial y} \cdot \frac{\partial v}{\partial x}\right)=0$

qui est l'équation de la parabole dérivée.

Considérations géométriques sur les coniques dérivées. On constate facilemente que les points

$$
\begin{array}{ll}
M\left(\frac{\partial u}{\partial x}, \frac{\partial v}{\partial y}\right), & N\left(\frac{\partial u}{\partial x}, \frac{1}{\mu} \frac{\partial u}{\partial y}\right), \\
P\left(\frac{\partial v}{\partial y}-\nu \frac{\partial v}{\partial x}, \frac{\partial v}{\partial x}\right), & Q\left(\frac{\partial v}{\partial y}-\frac{\nu}{\mu} \frac{\partial u}{\partial y}, \frac{1}{\mu} \frac{\partial u}{\partial y}\right),
\end{array}
$$

situés dans les sommets d'un trapèze rectangle appartient à la conique dérivée avec la notable propriété que le point $M$ est indépendant du champ élu.

Quand le déterminant $\Delta$ du champ complexe varie avec continuité, la conique passe par déformation continuelle d'hyperbole et d'ellipse en parabole, avec la caractéristique que le point $M$ demeure invariable.

La conique dérivée est générée par les faisceaux de droites

$$
\begin{aligned}
& Y=-\frac{1}{n \mu} X+\frac{1}{n \mu}\left(\frac{\partial u}{\partial x}+n \frac{\partial u}{\partial y}\right) \\
& Y=-\frac{n}{1+n \nu} X+\frac{1}{1+n \nu}\left(\frac{\partial v}{\partial x}+n \frac{\partial v}{\partial y}\right)
\end{aligned}
$$

qui passent par les points $P$ et $N$.

De plus on prouve que la conique est projective avec le faisceau selon lequel on détermine les dérivées radiaux.

Il est notable d'observer qu'il existe une ressemblance entre la figure du voisinage et la conique dérivée, de telle façon que à voisinage elliptique, paire de droites parallèles et voisinage hyperbole, une ellipse, une parabole et une hyperbole correspondent respectivement comme conique dérivée.

D'autre part, les équations (6), (7) et (8), quand on remplit les conditions de monogénéité, se transforment en paires de droites (imaginaires dans le champ complexe elliptique), dont l'interception est le dérivé holomorph dans le sens ordinaire.

A ce point de vue il existent infinis dérivés pour la fonction holomorphe, données par le point d'interception des droites (dérivé holomorph dans le sens ordinaire), et les infinies valeurs qui correspondent aux directions isotropes.

Cette interprétation est en parfaite harmonie avec le concept de 
point généralisé que nous venons d'exposer; de manière que nous pouvons dire encore que le dérivé holomorph d'une fonction de variable complexe binaire est un point elliptique pour la variable complexe ordinaire, un point parabolique pour le champ complexe parabolique et un point hyperbolique pour le champ complexe hyperbolique.

De la même façon la conique dérivée pour la fonction polygène est formée par points elliptiques, paraboliques et hyperboliques, selon le cas.

Quand on accomplit les conditions de monogénéité, la conique se déforme comme nous avons déjà dit, de manière que les points généralisés se transforment dans le point généralisé correspondant à le dérivé unique.

Remarque. Nous faisons noter que dans le champ complexe plus général de deux dimensions $a \alpha_{1}+b \alpha_{2}$ telle que $\alpha_{1}^{2}=\mu_{1} \alpha_{1}+\nu_{1} \alpha_{2} ; \alpha_{2}^{2}=\mu_{2} \alpha_{1}$ $+\nu_{2} \alpha_{2} ; \alpha_{1} \alpha_{2}=\alpha_{2} \alpha_{1}=\mu_{3} \alpha_{1}+\nu_{3} \alpha_{2}$, formellement, il résulte pour les fonctions polygènes $d u$ variable de ce champ la conique suivante

$$
\begin{aligned}
\left|\begin{array}{ll}
\mu_{1} & \nu_{1} \\
\mu_{2} & \nu_{2}
\end{array}\right| X^{2} & +\left|\begin{array}{ll}
\mu_{2} & \nu_{2} \\
\mu_{3} & \nu_{3}
\end{array}\right| Y^{2}+\left|\begin{array}{ll}
\mu_{1} & \nu_{1} \\
\mu_{3} & \nu_{3}
\end{array}\right| X Y \\
& -\left(\left|\begin{array}{ll}
\mu_{1} & v_{x} \\
\mu_{2} & v_{y}
\end{array}\right|-\left|\begin{array}{cc}
\nu_{1} & u_{x} \\
\nu_{2} & u_{y}
\end{array}\right|\right) X \\
& -\left(\left|\begin{array}{ll}
\mu_{2} & v_{x} \\
\mu_{3} & v_{y}
\end{array}\right|-\left|\begin{array}{cc}
\nu_{2} & u_{x} \\
\nu_{3} & u_{y}
\end{array}\right|\right) Y+\left|\begin{array}{ll}
u_{x} & v_{x} \\
u_{y} & v_{y}
\end{array}\right|=0
\end{aligned}
$$

qui contient toutes les antérieures comme des cas particuliers.

Je me limite à ces résultats fondamentaux des fonctions polygènes du nombre binaire, pour ne pas m'étendre trop sur cela, mais je vais indiquer seulement quelques résultats, sans démonstration pour ne pas entrer en détails.

Représentation conforme. Nous définissons la représentation conforme dans le champ binaire général comme étant une correspondance biunivoque qui conservent les arguments précédemment définis, c'està-dire, dans le sens de conservation d'aire. Nous démontrons que dans le champ complexe général on conserve la relation fondamentale entre l'holomorphisme et la représentation conforme, c'est-à-dire, que toute fonction holomorphe conserve les arguments.

Fonction linéaire (de Moebius). Nous faisons l'étude analogue au classique sur la fonction $w=(a z+b) /(c z+d)$ démontrant que le résultat classique de la conservation de circonférences subsiste dans le champ général. 
Or, nous démontrons que la fonction linéaire change la conique en conique (hyperboles dans le champ complexe hyperbolique; droites dans le champ parabolique et ellipses dans le champ elliptique) et réciproquement (données deux coniques il existe une fonction linéaire qui les fait correspondre)-à la condition que ces coniques soient homo-asymptotiques et des asymptotes parallèles aux droites isotropes.

Cette restriction apparente n'existe pas, parce que dans le champ classique toutes les circonférences sont homo-asymptotiques.

J'ai étudié également quelques autres sujets ayant rapport avec la fonction linéaire.

Buenos Aires, Argentina 